\title{
APLICACIÓN DE UN PROGRAMA DE CREACIÓN DE PROBLEMAS PARA MEJORAR LA COMPETENCIA MATEMÁTICA
}

\author{
APPLICATION OF A POSING PROBLEM PROGRAME TO \\ IMPROVE MATHEMATICAL COMPETENCE
}

Gilberto Platero Aratia ${ }^{1}$, Lupita Esmeralda Arocutipa Huanacuni ${ }^{2}$, Bandalia Sarai Antiquera Nina ${ }^{3}$

\section{RESUMEN}

El presente estudio examina la competencia matemática mediada por un programa de creación de problemas con el objetivo de mejorar la competencia de docentes del área de matemática. Para ello, se consideró como unidad de estudio una muestra intacta de 74 docentes de educación secundaria en ejercicio de la ciudad de Tacna (Perú), a quienes se les implementó un diseño cuasiexperimental con grupo control y experimental. El programa plantea 12 talleres de trabajo en torno a 3 fases que representan las partes fundamentales del proceso de creación de problemas matemáticos.

Los resultados muestran un incremento del nivel de competencia matemática de docentes del grupo experimental. De los $27,8 \%$ ubicados en el nivel de competencia significativo - nivel 2 y 3 - (antes de aplicar el programa) pasaron al 63,9\% (después de aplicar el programa). En consecuencia, la aplicación del programa de creación de problemas mejora significativamente la competencia matemática de los docentes del grupo experimental, con un nivel de significancia del $5 \%$.

Palabras clave: Creación de problemas, competencia matemática, resolución de problemas.

\begin{abstract}
The present study examines the mathematical competence mediated by a problem-posing program with the aim of improving the competence of teachers in the area of mathematics. For this purpose, an intact sample of 74 teachers of secondary education in the city of Tacna (Perú) was studied as a unit of study, who were given a quasi-experimental design with a control and experimental group. The Program presents 12 workshops around 3 phases that represent the fundamental parts of the process of posing mathematical problems.

The results show an increase in the level of mathematical competence of teachers in the experimental group. Of the $27.8 \%$ teachers located at the level of significant competition-level 2 and 3- (before implementing the program) went up to $63.9 \%$ (after applying the program). Consequently, the application of the problem-posing program significantly improves the mathematical competence of teachers in the experimental group, with a significance level of $5 \%$.
\end{abstract}

Keywords: Problems posing, mathematical competence, problem

\footnotetext{
${ }^{1}$ Universidad Nacional Jorge Basadre Grohmann. Tacna-Perú. E-mail:gilbertoplatero@gmail.com.

${ }^{2}$ Universidad Nacional Jorge Basadre Grohmann. Tacna-Perú. E-mail:lupitaeah@gmail.com.

${ }^{3} \mathrm{IEPM}$ “Crl. Gregorio Albarracín Lanchipa”UGEL Tacna. Tacna-Perú. E-mail: bandaliasarai@gmail.com
} 


\section{INTRODUCCIÓN}

Los bajos resultados en pruebas nacionales e internacionales, en relación con las competencias matemáticas de estudiantes que terminan la educación básica regular, así como de profesores en formación y en ejercicio - OCDE (2013), OCDE (2016) y Díaz (2015) - motivó el desarrollo del presente estudio que gira en torno a la pregunta ¿cuál es el grado de eficacia del programa de creación de problemas para mejorar la competencia matemática en docentes de educación secundaria de Tacna? Así, se propone un programa de creación de problemas, basado en los aportes teóricos de Fernández \& Barbarán (2015) y Malaspina (2013), que estimula a los docentes al uso de tareas que involucren actividades de creación de problemas para mejorar sus competencias matemáticas. E1 docente que logre desarrollar la capacidad de crear problemas de matemáticas podrá plantear a sus alumnos problemas adecuados, según el contexto donde se desenvuelven, fomentando en ellos la creación de sus propios problemas como parte de su aprendizaje.

Estudios realizados por Martínez (2015), Torres (2016), Cruz (2002), Salazar (2014) y Fernández (2010) muestran que el uso de estrategias de creación de problemas favorece la creatividad, el pensamiento matemático y la competencia matemática. Pero ¿qué se entiende por crear problemas de matemáticas y por competencia matemática? Para Silver (1994), "crear problemas se refiere a la generación de nuevos problemas y a la re-formulación de problemas dados. Por lo tanto, este proceso puede ocurrir antes, durante, o después de la resolución de un problema" (p.19). Desde la perspectiva de Malaspina (2013), "la creación de problemas matemáticos es un proceso mediante el cual se obtiene un nuevo problema a partir de un problema conocido (variación de un problema dado) o a partir de una situación dada (elaboración de un problema)" (p.131). La incorporación de estos procesos en la práctica pedagógica permite al docente implementar tareas con situaciones cercanas al entorno de sus estudiantes, facilitando así su aprendizaje.
En actividades de creación de problemas es fundamental saber distinguir los elementos que forman parte de un problema de matemática. Según Malaspina (2013) y Malaspina \& Vallejo (2014), estos elementos son: la información, el requerimiento, el contexto y el entorno matemático. La información se refiere a los datos cuantitativos o relacionales que se dan en el problema. El requerimiento es lo que se pide que se encuentre, examine o concluya -cuantitativo o cualitativo-, incluyendo gráficos y demostraciones.

El contexto puede ser intra matemático o extra matemático. En el primer caso, el problema se circunscribe a lo matemático; por ejemplo, hallar el dominio de una función, graficar una ecuación de dos variables y demostrar la desigualdad triangular para números reales: resulta evidente que son problemas con un contexto intra matemático. En el segundo caso, el problema está más vinculado a una situación real por lo que suele llamarse problema contextualizado. Finalmente, el entorno matemático se refiere a los conceptos matemáticos que intervienen o pueden intervenir para resolver el problema, por tanto está supeditado al camino que se siga para resolver el problema.

En esa dirección, Malaspina (2013) y Malaspina \& Vallejo (2014) establecen que la creación de un problema de matemáticas, como variación de un problema dado, es un proceso según el cual se construye un nuevo problema modificando la información, el requerimiento, el contexto o el entorno matemático; es decir, modificando uno o más de los cuatro elementos del problema inicial. Respecto a la creación de un problema de matemáticas es la elaboración de un problema a partir de una situación específica. En consecuencia, estos autores la definen como el proceso mediante el cual se construye un problema cuyo contexto es tal situación $u$ otra inspirada por ella. Estos datos son obtenidos por selección o modificación de la información que se percibe en la situación dada; cuyo requerimiento es factible mediante relaciones lógicas y matemáticas establecidas o encontradas entre los elementos de la información especificada, que están implícitas en el enunciado, dentro de un cierto entorno 
matemático. Por lo tanto, cuando se enfrenta a actividades de creación de problemas, es fundamental saber identificar los elementos del problema y conocer el dominio conceptual en relación a este; ello permitirá crear nuevos problemas, ya sea por variación de uno dado o por elaboración de una situación específica. Sin embargo, como afirma Polya (1965), "encontrar un nuevo problema que sea a la vez interesante y accesible no es fácil; se necesita experiencia, buen gusto y suerte" (p. 171).

Referente a la noción de competencia matemática, Niss (2002) plantea que la competencia matemática es la "habilidad para entender, juzgar, hacer y usar las matemáticas en una variedad de situaciones y contextos intra y extra matemáticos, en los que las matemáticas juegan o podrían jugar un papel" (p.7). Una de las definiciones de mayor difusión y aceptación de competencia matemática en los últimos años es la propuesta dada por la OECD (2013):

La capacidad del individuo para formular, emplear e interpretar las matemáticas en distintos contextos. Incluye el razonar matemáticamente y la utilización de conceptos, procedimientos, datos y herramientas matemáticas para describir, explicar y predecir fenómenos. Ayuda a los individuos a reconocer el papel que las matemáticas desempeñan en el mundo y a emitir los juicios y las decisiones bien fundadas que necesitan los ciudadanos constructivos, comprometidos $\mathrm{y}$ reflexivos.(p.17)

Formular implica encontrar situaciones a través de la observación e imaginación que generen oportunidades para aplicar y utilizar las matemáticas. Emplear tiene que ver con el uso de razonamientos matemáticos, conceptos, procedimientos, datos y herramientas matemáticas a fin de resolver problemas de tipo intra o extra matemáticos. Interpretar supone reflexionar sobre soluciones matemáticas e interpretarlos en el entorno del problema o situación presentada. Las definiciones formuladas destacan el dominio funcional de la matemática; es decir, el uso de la matemática en contextos reales. Esto significa que el docente debe enfocar el trabajo sobre la resolución de problemas que involucren situaciones cotidianas según el entorno social y cultural de los estudiantes; favoreciendo así, el desarrollo de la competencia matemática de los mismos. Sin embargo, no es posible que el docente canalice un proceso de aprendizaje orientado al dominio funcional de la matemática, si él mismo no posee esas competencias.

Las limitaciones que se presentaron en el desarrollo del presente estudio fueron las escasas investigaciones respecto a la creación de problemas y la competencia matemática de profesores en ejercicio y profesores en formación. Por lo demás, no se encontró limitaciones humanas, materiales ni económicas significativas que afecten el desarrollo de esta investigación; puesto que se contó con recursos económicos y materiales, el apoyo de las autoridades de la institución, tanto como la aceptación de los participantes.

Por las razones planteadas, pretendemos demostrar que la aplicación del programa de creación de problemas mejora la competencia matemática en docentes de educación secundaria de Tacna.

\section{Hipótesis}

La aplicación del programa de creación de problemas mejoraría significativamente la competencia matemática en docentes de educación secundaria de Tacna.

\section{MATERIALES Y MÉTODOS}

Se utilizó un diseño cuasiexperimental con dos grupos (control y experimental). Ambos grupos fueron evaluados antes y después de la aplicación del programa de creación de problemas matemáticos. La muestra fue no probabilística y por conveniencia, constituida por 74 docentes de matemática de educación secundaria de Tacna, 38 asistentes del proyecto de innovación pedagógica de la Unidad de Gestión Educativa Local y 36 asistentes del diplomado Creación de problemas matemáticos realizado en la Escuela 
Profesional de Educación de la Universidad Nacional Jorge Basadre Grohmann. Los docentes del proyecto de innovación pedagógica constituyeron el grupo control y los asistentes del diplomado, el grupo experimental.

La variable independiente fue el programa de creación de problemas, validada mediante criterio de jueces por tres especialistas. Tuvo el propósito de estimular la capacidad de creación de problemas en docentes o alumnos; por ello, giró en torno a tres fases que representan una parte fundamental del proceso de creación. La variable dependiente fue la competencia matemática, medida con la prueba de desarrollo (pretest-postest) que es denominada "prueba para medir el nivel de competencia matemática". Dicho instrumento (compuesto por 40 ítems y diseñado para evaluar las siguientes competencias: pensar y razonar, plantear y resolver problemas, modelizar, argumentar, representar y comunicar) fue validado a través de criterio de jueces por tres especialistas y posteriormente se llevó a la práctica como prueba piloto, lo cual permitió establecer la confiabilidad de la prueba por medio del coeficiente de estabilidad; 0,955 para el grupo control y 0,915 para el experimental, los cuales indican una buena precisión del instrumento. La variable interviniente, controlada a través de una ficha de registro, fue la asistencia a clases.

Tabla 1

Escala de medición de la competencia matemática.

\begin{tabular}{ccc}
\hline \hline $\begin{array}{c}\text { COMPETENCIA } \\
\text { MATEMÁTICA }\end{array}$ & $\begin{array}{c}\text { ESCALA DE } \\
\text { MEDICIÓN }\end{array}$ & \multicolumn{1}{c}{ DESCRIPCIÓN } \\
\hline Nivel 3 & 347 a más & $\begin{array}{l}\text { Conceptualizan, generalizan y utilizan la información y modelos de situaciones } \\
\text { de problemas complejos. Son capaces de pensar y razonar con matemática avanzada. } \\
\text { Formulan y comunican con precisión sus acciones y reflexiones referidas a sus } \\
\text { resultados, interpretaciones y argumentos, y su pertinencia a situaciones originales. }\end{array}$
\end{tabular}

\begin{tabular}{lll}
\hline Nivel 2 & 276 a 346 & $\begin{array}{l}\text { Desarrollan y trabajan con modelos de situaciones concretas y complejas. Seleccionan, } \\
\text { comparan y evalúan estrategias de resolución de problemas para abordar problemas } \\
\text { relacionados con estos modelos. Reflexionan sobre sus acciones y pueden formular } \\
\text { y comunicar sus interpretaciones y razonamientos. }\end{array}$ \\
\hline Nivel 1 & 205 a 275 & $\begin{array}{l}\text { Ejecutan procedimientos claramente descritos. Sus interpretaciones son sólidas para } \\
\text { seleccionar y aplicar estrategias de solución de problemas sencillos. También interpretan } \\
\text { y utilizan representaciones basadas en diferentes fuentes de información y razonan } \\
\text { directamente a partir de ellos. }\end{array}$
\end{tabular}

Debajo del nivel 1 Menos de 205 Evidencia dificultades para realizar las tareas de matemáticas más elementales que se le pide.
Inicialmente, en el mes de agosto de 2015, se aplicó la prueba para medir el nivel de competencia matemática (pretest), simultáneamente al grupo control y experimental. Durante el proceso, se realizó una presentación didáctica al grupo experimental respecto a la creación de problemas y a los elementos que se perciben en un problema (información, requerimiento, contexto y entorno matemático) y que la modificación adecuada de uno o más de estos elementos generan nuevos problemas. Luego se dio a conocer algunas ventajas de la importancia de crear problemas en las clases de matemáticas y se mostraron varios ejemplos didácticos sobre creación de problemas por variación y elaboración según el nivel educativo requerido. Se desarrollaron 12 talleres (de 100 minutos aproximadamente, durante 3 meses) de trabajo individual y grupal según las fases de creación de problemas consideradas en el programa. Finalmente, a los docentes se les aplicó la prueba para medir el nivel de competencia matemática (postest), evento que se realizó en el mes de noviembre de 2015. Las puntuaciones obtenidas permitieron establecer, a través de cuadros de distribución de frecuencias, los niveles de competencia matemática. Las hipótesis se comprobaron utilizando la prueba de la distribución normal $\mathrm{Z}$ para proporciones y para datos apareados, con un nivel de significancia del $5 \%$. Los medios informáticos empleados fueron Microsoft Excel y SPSS. 


\section{RESULTADOS}

Los resultados muestran que no existen diferencias significativas en los niveles de competencia antes de aplicar el programa de creación de problemas.

Tabla 2

Niveles de competencia matemática, según puntuaciones del pretest.

\begin{tabular}{lccccc}
\hline \hline \multirow{2}{*}{ Niveles } & \multicolumn{2}{c}{ Grupo control } & & \multicolumn{2}{c}{ Grupo experimental } \\
\cline { 2 - 3 } \cline { 5 - 6 } & Frecuencia & Porcentaje & & Frecuencia & Porcentaje \\
\hline < nivel 1 & 2 & 5,3 & & 6 & 16,7 \\
Nivel 1 & 28 & 73,6 & & 20 & 55,5 \\
Nivel 2 & 8 & 21,1 & & 10 & 27,8 \\
Nivel 3 & 0 & 0,0 & & 0 & 0,0 \\
Total & 38 & 100,0 & & 36 & 100,0 \\
\hline \hline
\end{tabular}

Después de aplicar el programa de creación de problemas, se evidenció que existe un mejor nivel de logro de competencias en el grupo experimental que en el grupo control, lo cual fue verificado con la prueba $\mathrm{Z}$ para datos apareados $(\mathrm{Zc}=15,16>1,96)$. Por tanto, se rechaza la hipótesis nula de que no hay diferencias de nivel de competencias con un margen de error del $5 \%$.

Tabla 3

Niveles de competencia matemática, según puntuaciones del postest.

\begin{tabular}{lccccc}
\hline \multirow{2}{*}{ Niveles } & \multicolumn{2}{c}{ Grupo control } & & \multicolumn{2}{c}{ Grupo experimental } \\
\cline { 2 - 3 } \cline { 5 - 6 } & Frecuencia & Porcentaje & & Frecuencia & Porcentaje \\
\hline < nivel 1 & 2 & 5,3 & & 0 & 0,0 \\
Nivel 1 & 25 & 65,8 & & 11 & 30,5 \\
Nivel 2 & 11 & 28,9 & & 23 & 63,9 \\
Nivel 3 & 0 & 0,0 & & 2 & 5,6 \\
$\quad$ Total & 38 & 100,0 & & 36 & 100,0 \\
\hline \hline
\end{tabular}

Respecto a la media y considerando una valoración de 0 a 400 puntos, en el pretest se evidenció que hay similitud de promedios en ambos grupos de estudio (247,9 para el grupo control y 245,1 para el experimental). Sin embargo, en el postest se percibió una diferencia de promedios a favor del grupo experimental (282) frente al grupo control $(254,9)$.

En consecuencia, se comprobó que la aplicación del programa de creación de problemas mejora significativamente la competencia matemática de los docentes del grupo experimental, rechazándose la hipótesis nula de que la competencia matemática después de aplicar el programa, se mantiene con el $5 \%$ de nivel de significancia.

Tabla 4

Contrastación de la hipótesis principal del estudio.

\begin{tabular}{|c|c|c|c|c|}
\hline Hipótesis nula y alternativa & Estadígrafo & $\begin{array}{l}\text { Zona de } \\
\text { rechazo }\end{array}$ & $\begin{array}{c}\text { Valor } \\
\text { calculado }\end{array}$ & Decisión \\
\hline $\begin{array}{l}\text { Ho: La competencia matemática, } \\
\text { después de aplicar el programa } \\
\text { de creación de problemas, se } \\
\text { mantiene. ( } \mu \text { después }=\mu \text { antes) } \\
\text { Ha: La competencia matemática, } \\
\text { después de aplicar el programa } \\
\text { de creación de problemas, ha } \\
\text { mejorado. ( } \mu \text { después }>\mu a n t e s)\end{array}$ & $\begin{array}{l}\text { Prueba } \mathrm{Z} \\
\text { para datos } \\
\text { apareados } \\
\mathrm{Zc}=\frac{\overline{\mathrm{D}}}{S_{D} / \sqrt{n}}\end{array}$ & {$[1,65 ; \infty)$} & $\mathrm{Zc}=15,16$ & $\begin{array}{l}\mathrm{Zc} \in[1,65 ; \infty\rangle \\
\text { entonces } \\
\text { se rechaza Ho }\end{array}$ \\
\hline
\end{tabular}

Nivel de significancia: $\alpha=0,05$ 


\section{DISCUSIÓN}

Ante los resultados bajos del desempeño académico de los estudiantes, surge la siguiente pregunta: ¿será posible que docentes que no son matemáticamente competentes puedan formar a estudiantes que sí lo sean? En este sentido, los hallazgos cuantitativos obtenidos a lo largo de la investigación convergen para el logro de docentes matemáticamente competentes, mediado a través de la aplicación de un programa de creación de problemas matemáticos.

Desde esa perspectiva, los resultados obtenidos con las puntuaciones del pretest aplicado a los docentes del grupo control muestran un promedio de 247,9 y los docentes del grupo experimental, un promedio de 245,1; es decir, antes de aplicar el programa de creación de problemas, ambos grupos se hallan en promedio en el nivel de competencia 1 , esto implica que los docentes solo logran ejecutar procedimientos claramente descritos, sus interpretaciones son sólidas para seleccionar y aplicar estrategias de solución de problemas sencillos; aunque también interpretan y utilizan representaciones basadas en diferentes fuentes de información y razonan directamente a partir de ellos. Estos resultados están en correspondencia con los reportes elaborados por Díaz (2015) y Torres (2016), según los cuales se perciben dificultades en la comprensión y el desempeño académico de los docentes en el área de matemática. Por ende, es necesario que los currículos de formación docente incorporen tareas que conlleven a mejorar sus competencias no solo profesionales; sino, además, sus competencias matemáticas bajo una concepción constructivista del aprendizaje. Los resultados permitieron diseñar y aplicar un programa de creación de problemas, orientado a mejorar la competencia matemática de docentes.

Los resultados del postest evidencian que el nivel de competencia matemática ha mejorado significativamente en el grupo experimental, incrementándose de 245,1 a 282. Además, el porcentaje de docentes ubicados en el nivel 1 es $65,8 \%$ significativamente mayor en el grupo de control, con respecto al grupo experimental, que resultó de $30,5 \%$. En el grupo experimental $63,9 \%$ logró ubicarse en el nivel 2, lo cual implica que los docentes de este nivel de competencia desarrollan y trabajan con modelos de situaciones concretas y complejas; seleccionan, comparan y evalúan estrategias de resolución de problemas para abordar problemas relacionados con estos modelos; reflexionan sobre sus acciones y pueden formular sus interpretaciones $y$ razonamientos. Asimismo, un 5,6\% logró ubicarse en el nivel 3; es decir, estos docentes conceptualizan, generalizan y utilizan la información sobre la base de sus investigaciones y modelos de situaciones de problemas complejos; son capaces de pensar y razonar con matemática avanzada; formulan y comunican con precisión sus acciones y reflexiones referidas a sus resultados, interpretaciones y argumentos, y su pertinencia a situaciones originales. Ello permite evidenciar la eficacia del programa de creación de problemas para mejorar la competencia matemática. Estas afirmaciones son respaldadas por investigaciones realizadas por Cruz (2002), Castro (2011), Cárdenas (2015), Salazar (2014), Fernández (2010) y Malaspina (2013); quienes comprobaron la efectividad del uso de estrategias de creación de problemas para mejorar la creatividad y el pensamiento matemático de los estudiantes.

Para contrastar las hipótesis se aplicaron las pruebas paramétricas, dado que los datos analizados siguen una distribución normal. Así se demostró, con un margen de probabilidad del $5 \%$, que la aplicación del programa de creación de problemas mejora significativamente la competencia matemática de los docentes de educación secundaria de Tacna (del grupo experimental). Con ello, se corroboraron los estudios realizados por Torres (2015), Arreguín (2009), Salazar (2014) y Fernández (2010); quienes establecieron que las actividades orientadas a fomentar la capacidad de creación de problemas matemáticos inciden positivamente en la comprensión y rendimiento académico de los sujetos; por tanto, desarrolla la competencia matemática de estos. Cabe 
destacar que los docentes valoraron positivamente el programa aplicado; pues además de fomentar sus capacidades innatas de creación de problemas, los ayudó significativamente a incrementar sus conocimientos matemáticos y a desarrollar su creatividad.

\section{CONCLUSIONES}

1. Se determinó que el nivel de competencia matemática de docentes, antes de aplicar el programa de creación de problemas es poco significativo; esto significa que la mayoría de docentes evaluados se ubicaron en el nivel 1 y por debajo del mismo $(72,2 \%)$, evidenciando procedimientos claramente descritos; además, sus interpretaciones son sólidas solo para seleccionar y aplicar estrategias de solución de problemas sencillos, aunque también interpretan y utilizan representaciones basadas en diferentes fuentes de información y razonan directamente a partir de ellos.

2. Se estableció que el nivel de competencia matemática de docentes, después de aplicar el programa de creación de problemas es significativo; puesto que la mayoría de docentes evaluados se ubicaron en el nivel $2(63,9 \%)$. Los docentes de este nivel de competencia desarrollan y trabajan con modelos de situaciones concretas y complejas; seleccionan, comparan y evalúan estrategias de resolución de problemas para abordar problemas relacionados con estos modelos; reflexionan sobre sus acciones y pueden formular sus interpretaciones y razonamientos.

3.Se comprobó que existe diferencia estadísticamente relevante del nivel de competencia matemática de docentes, antes y después de aplicar el programa de creación de problemas. Los docentes ubicados en el nivel de competencia significativo pasaron del $27,8 \%$ (antes de aplicar el programa) al 63,9\% (después de aplicar el programa).

4. Se demostró que la aplicación del programa de creación de problemas mejora significativamente la competencia matemática en docentes de educación secundaria de Tacna (del grupo experimental), con un nivel de significancia del $5 \%$. Con lo cual se acredita la importancia de crear problemas para mejorar el nivel de competencia matemática.

\section{REFERENCLAS BIBLIOGRÁFICAS}

Arreguín, L. (2009). Competencias matemáticas usando la técnica de aprendizaje orientado en proyectos (Tesis de maestría). Tecnológico de Monterrey, Universidad Virtual, Escuela de Graduados en Educación, México.

Cárdenas, J. (2015). Análisis de problemas de adición, sustracción y multiplicación de expresiones decimales, creados por estudiantes del $6^{\circ}$ grado de primaria en una experiencia didáctica (Tesis de maestría). Pontificia Universidad Católica del Perú, Lima.

Castro, E. (2011). La invención de problemas y sus ámbitos de investigación. SEIEM. Investigaciones en pensamiento numérico y algebraico e historia de la matemática y educación matemática. Universidad de Granada, España. Recuperado de http://www.seiem.es/gruposdetr abajo/pna/ActasPNAGranada. pdf

Cruz, M. (2002). Estrategia metacognitiva en la formulación de problemas para la enseñanza de la matemática (Tesis doctoral). Instituto Superior Pedagógico de Holguín, Cuba.

Díaz, H. (2015). Formación docente en el Perú. Realidades y tendencias. Lima: Santillana.

Fernández, J. (2010). La invención de problemas y el desarrollo de la competencia matemática. EduPsykhé, 9(2), 221-234.

Fernández, J. y Barbarán, J. (2015). Inventar problemas para desarrollar la competencia matemática. Madrid: La Muralla, S.A. 
Malaspina, U. (setiembre, 2013). La creación de problemas matemáticos en la formación de profesores. VII CIBEM. Simposio llevado a cabo e $n$ e 1 V I I C o n gre so Iberoamericano de Educación Matemática, Montevideo, Uruguay. Recuperado de http://www.cibem7.semur.edu.u y/7/actas/pdfs/729.pdf

Malaspina, U., y Vallejo, E. (2014). Creación de problemas en la docencia e investigación. En Reflexiones y Propuestas en Educación Matemática (pp. 7-54). Lima, Perú: Editorial Moshera S.R.L.

Martínez, C. (2015). Estrategias para estimular la creación de problemas de adición y sustracción de números naturales con profesores de educación primaria (Tesis de maestría). Pontificia Universidad Católica del Perú, Lima.

Niss, M. (2002). Mathematical competencies and the learning of mathematics: The Danish Kom Project. $R$ e c u p e r a d o d e http://equipsice.uab.cat/file.php/ 28/Compet_Niss.pdf

OCDE (2013). Informe de resultados PISA (Programa para la Evaluación Internacional de Estudiantes) 2012. Re cuperado de http://www.oecd.org/pisa/keyfin dings/ PISA-2012-resultsmexico-ESP.pdf

OCDE (2016). Informe de resultados PISA 2015 . Recuperado de https://www.oecd.org/ pisa/pisa2015-results-in-focus-ESP.pdf

Polya, G. (1965). Cómo plantear y resolver problemas. México: Trillas.

Salazar, L. (2014). Creación de problemas como recurso didáctico en cursos de matemática formal dirigidos a futuros profesores de matemática de secundaria. CIDUI. Revista del Congrés Internacional de Docència Universitària i Innovació. Universidad de Costa Rica. Recuperado de http://www.cidui.org/ revistacidui/index.php/cidui/ article/view/543/524

Silver, E. (1994). On Mathematical Problem Posing. For the Learning of Mathematics, 14(1), 19-28. Recuperado de http://flmjournal.org/Articles/2A5D1527 $\begin{array}{lllll}7 & 8 & 1 & 4 & 1\end{array}$ F58C1966ED8673C15.pdf

Torres, C. (2016). Creación de problemas sobre funciones cuadráticas por profesores en servicio, mediante una estrategia que integra nociones del análisis didáctico (Tesis de maestría). Pontificia Universidad Católica del Perú, Lima. 